\section{The effect of COVID-19 emergency in the management of melanoma in Italy}

\author{
Intergruppo Melanoma Italiano*
}

\begin{abstract}
The COVID-19 pandemic has severely hampered the functioning of any health system, absorbing a considerable amount of resources and with the threat of widespread infection in the health services. The present survey has been carried out in Italy to evaluate if and how COVID-19 also affected skin melanoma management. We enrolled 13 Italian centres highly qualified in the diagnosis and care of skin melanoma. We compared a set of information evaluating the amount of activity for melanoma performed during February-April 2020 with the same quarter in 2019. The number of new melanoma diagnosis, biopsies, wide local excisions, overall pathology reports decreased. However, the most severe cases seem promptly managed with sentinel lymph node biopsies, new systemic treatments (north) and the total number of (advanced) treated patients (centre-south). The COVID-19 experience has underlined the need to exploit the help which may come from telemedicine.
\end{abstract}

\section{Introduction}

The COVID-19 pandemic has caused, in addition to the specific effects, also a significant obstacle to the normal functioning of Health Services not directly related to the diagnosis and treatment of these patients. This effect due to rules to avoid infection spread in the health sector has recently been underlined, for its globality and severity, also by the World Health Organization. ${ }^{1}$

Substantially all types of services have suffered this backlash from the pandemic, with negative consequences for patients, even those suffering from serious diseases. The WHO states that $42 \%$ of the 155 countries investigated reduced or stopped the services aimed at cancer patients due to Codiv-19. In Italy, the first two cases of COVID-19 occurred on January $23^{\text {rd }}$. Subsequently, the infection spread with high intensity, especially in some northern regions, leading to measures of severe social distancing maintained from March $9^{\text {th }}$ to May $18^{\text {th }} 2020 .^{2}$ Such actions caused an almost $80 \%$ blockade of general dermato- logical activities in both public and private sector. ${ }^{3}$ Some dermatologic centres implemented rules for the assessment of the presence/absence of SARS2 infection in patients, prioritising their care according to the severity of diseases. ${ }^{4}$ We aimed at evaluating the extent of a COVID-19 effect in Italy on the activity of a series of services dedicated to the diagnosis and treatment of skin melanoma by comparing an epidemic period with a previous one.

\section{Material and Methods}

The following Italian centres were involved: the Hospital of national importance and high specialization (ARNAS) Garibaldi of Catania, the Istituto Fisioterapico Ospedaliero (IFO) of Rome, the AOU of Sassari, the Medical Oncology of University of Bari, the IRCCS Tumour Institute "Giovanni Paolo II" of Bari, the IRCSS IRST Romagna, the University of Parma, the Department of Pathological Anatomy of AUSL Romagna, the National Cancer Institute of Naples, the University of Florence, the Dermatological Clinic of the University of Turin, the IRCCS Foundation the National Cancer Institute of Milan and the Immuno-Oncology Centre-University Hospital of Siena.

A quarter (February-April) preceding the pandemic (relating to the year 2019) and one in the pandemic (the year 2020) were considered.

For both periods the participating centres were asked to collect data relating to: number of first visits; number of biopsies performed; number of wide local excisions; number of sentinel lymph node biopsies; number of new systemic medical therapies; number of total patients being treated.

The data concerned with cases before melanoma diagnosis: first visits, biopsies; cases with a positive biopsy for melanoma: wide local excision; cases with a positive biopsy for melanoma and indication for lymph node evaluation: sentinel node biopsy; cases of newly or previously diagnosed melanoma with advanced stage or evolved from earlier stages: the beginning of systemic medical therapies; finally, the total number of patients (with advanced melanoma) in therapy.

The participating centres are in the northern Italian regions, most affected by the pandemic (Lombardy, Emilia Romagna, and Piedmont) and in central (Tuscany and Lazio) and southern regions (Sicily, Puglia, and Sardinia) where the number of cases was sensibly lower.

The comparison of the extent of the cases between 2019 and 2020 was made
Correspondence: Ignazio Stanganelli, Skin Cancer Unit, Istituto Scientifico Romagnolo per lo Studio e la Cura dei Tumori (IRST), Istituto di Ricovero e Cura a Carattere Scientifico (IRCCS), Meldola, via Maroncelli, 40, Meldola (FC), Italy.

Tel.: + 39.543739100.

E-mail: ignazio.stanganelli@irst.emr.it

Key words: Melanoma; COVID-19; Italy; management.

Acknowledgements: The Intergruppo Melanoma Italiano is particularly grateful for the assistance given by Emanuele Crocetti.

Conflict of interest: the authors declare no potential conflict of interests.

Funding: none.

Availability of data and materials: all data and materials are available by authors.

Please cite this article as: Intergruppo Melanoma Italiano. The effect of COVID-19 emergency in the management of melanoma in Italy. Dermatol Rep 2021;13:8972.

Received for publication: 1 October 2020.

Accepted for publication: 28 October 2020.

This work is licensed under a Creative Commons Attribution-NonCommercial 4.0 International License (CC BY-NC 4.0).

${ }^{\circ}$ Copyright: the Author(s), 2021

Licensee PAGEPress, Italy

Dermatology Reports 2021; 13:8972

doi:10.4081/dr:2021.8972

assuming that the observed events had a cumulative distribution of Poisson.

\section{Results}

In Italy (Table 1) during February-April 2020 we observed, a substantial reduction ($31.3 \%$ ) in the number of first visits, in the number of biopsies (-36.5\%) and wide local excisions (-22.9\%) in comparison to 2019. Total histological diagnoses also decreased by about a quarter, and the new therapies dropped by about a fifth $(-20.8 \%)$. On the contrary, no decrease in the sentinel node biopsies count and a reduction, within the limits of significance, in the overall number of patients currently being treated was observed.

By evaluating the northern and centralsouthern services separately, we observed a homogeneous reduction in the number of 
first visits, biopsies, wide local excisions and total histological diagnoses. In both areas, the number of sentinel lymph node biopsies did not decrease. Notably, there were two differences: the number of new systemic therapies did not reduce significantly in the north but did so $(-23.7 \%)$ in the centre-south, and the total number of patients under treatment remained stable in the centre-south, while it shrank in the north $(-14.0 \%)$.

\section{Discussion}

This survey had the aim of quantifying the effect of the COVID-19 pandemic in Italy in the management of cutaneous melanoma. The participating centres allowed comparisons of thousands of observations to provide a robust evaluation.

The comparison between the FebruaryApril 2019 and 2020 quarters takes for granted some assumptions:

i. the stability of the population (from which cases originate). In 2019 the Italian resident population counted up to $60,359,546$ inhabitants, according to National Institute of Statistics and, to $60,362,432$ in 2020 (www.demoistat.it);

ii. the incidence of melanoma is not decreasing; 5

iii. that stability in the two periods of the staff and the overall work capacity of the participating Services.

With this premise, we can affirm that in Italy - during the COVID-19 epidemic - we observed a substantial reduction in some of the elective activities of a group of highly specialised centres for cutaneous melanoma. In particular, there was a contraction of first visits and, consequently, of the biopsies that follow a melanoma suspicion and the wide local excisions following the histological confirmation of the biopsy. In the same way, histological diagnoses have decreased, presumably according to the decline in general diagnostic clinical activity.

The diminution of first visits and the ensuing diagnostic process can determine a temporal delay in melanoma diagnosis and a stage shift leading to more advanced melanomas with a worse prognosis. This risk has been underlined by the scientific community, ${ }^{6-8}$ which has led to recommendations for optimising the resources, directing them more to the most severe forms. ${ }^{9}, 10$ Such strategies apply to both melanoma and non-melanoma skin cancer patients for whom standard ${ }^{11}$ and advanced ${ }^{12,13}$ clinical and instrumental synopsis is essential.

A Spanish study estimated that the diagnostic delay of three months, related to the blocking of the activities of the COVID emergency, resulted in the melanoma stage $\mathrm{T} 1(\leq 1 \mathrm{~mm})$, which represents the majority of diagnosed melanoma, a decrease in the percentage of early diagnosis from $40 \%$ to $27 \%$. On the contrary, the T4 stage ( $>4 \mathrm{~mm}$ ) doubled from $16 \%$ to $30 \%{ }^{14}$ This latter stage represents has an estimated average increase in Breslow thickness greater than $0.50 \mathrm{~mm}$ per month. ${ }^{15}$ In the present study, the analysed centres seem to have accomplished the need for optimising the available resources during the COVID emergency. There was no decrease in sentinel node biopsies, aimed at evaluating the possible lymph node extension in newly diagnosed melanoma with severe prognostic figures. Moreover, in the north, where the COVID19 epidemic was more devastating, there was a decrease in the number of patients being treated (mainly patients with more advanced forms of melanoma who need systemic therapy). Still, this decrease did not concern the treatment of new patients in the quarter 2020 (similar in number to 2019), pointing at the ability to treat timely the most severe patients. On the contrary, in the centre-south, there was a decrease in new therapies but not in the treated patients, which remained stable.

The reduction or closure of the hospital and/or private outpatient activities for the COVID emergency ${ }^{1,2}$ has indeed delayed dermatological triage for suspected melanoma. Likewise, also the access to general medicine clinics suffered a blockage or marked reduction, also due to the limitation or absence of adequate spacing and containment measures that prevented appropriate triage also for all cancer prevention activities. ${ }^{1,2}$

Telemedicine support would have been useful for consultation between general medicine clinics and dermatological structures. Oncological teledermatology has become part of clinical practice even if more evidence is needed. ${ }^{16}$ However, in Italy, such a technique is still in a pioneering stage, ${ }^{17}$ also due to technological shortages and medico-legal restrictions. ${ }^{18}$

During the COVID pandemic, an Australian randomised study showed the usefulness of teledermatoscopy managed directly by trained patients, who sent images of suspected lesions straight to the dermatological reference centre, reporting a sensitivity higher than $75 \%$ and a specificity above $87 \% .{ }^{19}$ In Italy, the Istituto Superiore di Sanità strongly recommended during the COVID emergency to strengthen the implementation of telemedicine services. Telemedicine can turn to be very useful for both providing health services and for monitoring people in quarantine, including those with chronic disease as invasive melanoma, who need continuity of care. ${ }^{20}$

\section{Conclusions}

In Italy, the COVID-19 epidemic significantly affected the management of skin melanoma. The number of new melanoma diagnosis decreased in February-April 2020 in comparison with 2019. This shrunk activity may bring about a delay in melanoma diagnosis. However, the most severe cases seem promptly managed with sentinel

Table 1. The number of analysed melanoma diagnosis and treatment actions, for a quarter in 2019 and 2020 , percentage variation in 2020 in comparison with 2019 , for Italy as overall and for north and centre-south Italy.

\begin{tabular}{|c|c|c|c|c|c|c|c|c|}
\hline \multirow[t]{2}{*}{ Actions } & \multicolumn{3}{|c|}{ Italy } & \multirow[b]{2}{*}{$\mathbf{P}$} & \multicolumn{2}{|c|}{ North } & \multicolumn{2}{|c|}{ Centre-South } \\
\hline & Feb-Apr 2019 & Feb-Apr 2020 & $\%$ & & $\%$ & $\mathbf{P}$ & $\%$ & $\mathbf{P}$ \\
\hline First visits & 6.711 & 4.613 & -31.3 & $<0.01$ & -37.0 & $<0.01$ & -25.6 & $<0.01$ \\
\hline Biopsies & 4.458 & 2.829 & -36.5 & $<0.01$ & -26.4 & $<0.01$ & -46.1 & $<0.01$ \\
\hline Wide local excision & 1.072 & 827 & -22.9 & $<0.01$ & -11.5 & $<0.01$ & -38.5 & $<0.01$ \\
\hline Sentinel lymph node biopsy & 376 & 400 & +6.4 & 0.90 & +4.2 & 0.72 & +8.2 & 0.89 \\
\hline Overall pathological diagnosis & 3.156 & 2.386 & -24.4 & $<0.01$ & -14.6 & $<0.01$ & -33.9 & $<0.01$ \\
\hline Start new systemic therapy & 409 & 324 & -20.8 & $<0.01$ & -12.7 & 0.10 & -23.7 & $<0.01$ \\
\hline Overall patients in therapy & 1.734 & 1.667 & -3.9 & 0.054 & -14.0 & $<0.01$ & +1.9 & 0.74 \\
\hline
\end{tabular}


lymph node biopsies, new systemic treatments (north) and an overall number of treated (advanced) patients (south) stable. The COVID-19 experience has underlined the need to exploit more the help which may come from technology (e.g. Teledermatoscopy).

* Intergruppo Melanoma Italiano: Andrea Anichini, Salvatore Asero, Melissa Bersanelli, Corrado Caracò, Vincenzo De Giorgi, Anna Maria Di Giacomo, Claudio Feliciani, Virginia Ferraresi, Paola Ghiorzo, Antonio M. Grimaldi, Michele Guida, Mario Mandalà, Daniela Massi, Simone Mocellin, Giuseppe Palmieri, Roberto Patuzzo, Maria Antonietta Pizzichetta, Roberto Ricci, Giulio Rossi, Pietro Quaglino, Paola Queirolo, Rebecca Senetta, Ignazio Stanganelli, Marco Tucci

\section{References}

1. WHO. COVID-19 significantly impacts health services for noncommunicable diseases. 1 June 2020 Available from: ht t p s : / / w w w . who.int/new s room/detail/01-06-2020-COVID-19-significantly-impacts-health-services-fornoncommunicable-diseases

2. Remuzzi A, Remuzzi G. COVID-19 and Italy: what next? Lancet 2020;395:12258.

3. Gisondi P, PIaserico S, Bordin C, et al. Cutaneous manifestations of SARS-CoV2 infection: a clinical update. J Eur Acad Dermatol Venereol 2020;34:2499-504.

4. Tagliaferri L, Di Stefani A, Schinzari G, et al. Skin cancer triage and management during COVID-19 pandemic. J Eur Acad Dermatol Venereol 2020;34:1136-9.
5. AIOM-Airtum I Numeri del Cancro in Italia 2019. Intermedia editore. 2019.

6. Quinlan C, Gill R, Murphy M. Increasing melanoma awareness amongst health and social care professionals in secondary care in an era of reduced skin cancer referrals due to COVID-19. Clin Exp Dermatol 2020;45:920-1.

7. Tejera-Vaquerizo A, Nagore E. Estimated effect of COVID-19 lockdown on melanoma thickness and prognosis: a rate of growth model. J Eur Acad Dermatol Venereol 2020;34:e351-e3.

8. Gomolin T, Cline A, Handler MZ. The danger of neglecting melanoma during the COVID-19 pandemic. J Dermatolog Treat 2020;31:444-5.

9. Baumann BC, MacArthur KM, Brewer JD, et al. Management of primary skin cancer during a pandemic: Multidisciplinary recommendations. Cancer 2020;126:3900-6.

10. Rossi E, Trakatelli M, Giacomelli L, et al. The COVID-19 outbreak in dermatologic surgery: resetting clinical priorities. J Eur Acad Dermatol Venereol 2020;34:e5435.

11. Argenziano G, Soyer HP, Chimenti S, et al. Dermoscopy of pigmented skin lesions: results of a consensus meeting via the Internet. J Am Acad Dermatol 2003;48:679-93.

12. Peccerillo F, Mandel VD, Di Tullio F, et al. Lesions Mimicking Melanoma at Dermoscopy Confirmed Basal Cell Carcinoma: Evaluation with Reflectance Confocal Microscopy. Dermatology 2019;235:35-44.

13. Stanganelli I, Longo C, Mazzoni L, et al. Integration of reflectance confocal microscopy in sequential dermoscopy follow-up improves melanoma detection accuracy. Br J Dermatol 2015;172:365-
71.

14. Tejera-Vaquerizo A, Cañueto J, Toll A, et al. Estimated Effect of COVID-19 Lockdown on Skin Tumor Size and Survival: An Exponential Growth Model. Actas Dermosifiliogr 2020;S00017310:30142-30143.

15. Liu W, Dowling JP, Murray WK, et al. Rate of growth in melanomas: characteristics and associations of rapidly growing melanomas. Arch Dermatol 2006;142:1551-8.

16. Bruce AF, Mallow JA, Theeke LA. The use of teledermoscopy in the accurate identification of cancerous skin lesions in the adult population: A systematic review. J Telemed Telecare 2018;24:7583.

17. Cazzaniga S, Castelli E, Di Landro A, et al. Mobile teledermatology for melanoma detection: Assessment of the validity in the framework of a population-based skin cancer awareness campaign in northern Italy. J Am Acad Dermatol 2019;81:257-60.

18. Pagliarello C, Stanganelli I, Fabrizi G, et al. Digital Dermoscopy Monitoring: Is it Time to Define a Quality Standard? Acta Derm Venereol 2017;97:864-5.

19. Janda M, Swetter SM, Horsham C, Soyer HP. Virtual melanoma checks during a pandemic. Br J Dermatol 2020;183:7523.

20. Gabbrielli F, Bertinato L, De Filippis G, et al. Interim provisions on telemedicine healthcare services during COVID-19 health emergency. Accessed on: April 13, 2020. Available from: https://www.iss.it/documents/20126/0/R apporto+ISS+COVID19+n. $+12+$ telemed icina.pdf/37b4b856-603a-76c1-1b85$5 \mathrm{ff} 9 \mathrm{c} 662 \mathrm{bbbb} ? \mathrm{t}=1586860608120$. 